PROCEEDINGS OF THE

AMERICAN MATHEMATICAL SOCIETY

Volume 129, Number 8, Pages 2197-2203

S 0002-9939(01)05819-1

Article electronically published on January 17, 2001

\title{
SYMMETRIC NUMERICAL SEMIGROUPS WITH ARBITRARY MULTIPLICITY AND EMBEDDING DIMENSION
}

\author{
J. C. ROSALES
}

(Communicated by Wolmer V. Vasconcelos)

\begin{abstract}
We construct symmetric numerical semigroups $S$ for every minimal number of generators $\mu(S)$ and multiplicity $\mathrm{m}(S), 2 \leq \mu(S) \leq \mathrm{m}(S)-1$. Furthermore we show that the set of their defining congruence is minimally generated by $\mu(S)(\mu(S)-1) / 2-1$ elements.
\end{abstract}

\section{INTRODUCTION}

A numerical semigroup is a subset $S$ of $\mathbb{N}$ closed under addition, containing the identity element of $\mathbb{N}$ and which generates $\mathbb{Z}$ as a group (here $\mathbb{N}$ and $\mathbb{Z}$ denote the set of nonnegative integers and the set of integers, respectively). From this definition we obtain (see 1] and [7]) the following two results:

- The set $\mathbb{N} \backslash S$ is finite. We refer to the greatest integer not belonging to $S$ as the Frobenius number of $S$ and denote it by $\mathrm{C}(S)$.

- The semigroup $S$ has a unique minimal system of generators $\left\{n_{0}<n_{1}<\right.$ $\left.\ldots<n_{p}\right\}$. We refer to the numbers $n_{0}$ and $p+1$ as the multiplicity and the embedding dimension of $S$ respectively. We denote them by $\mathrm{m}(S)$ and $\mu(S)$, respectively.

Let $F=\left\{a_{0} X_{0}+\ldots+a_{p} X_{p} \mid a_{0}, \ldots, a_{p} \in \mathbb{N}\right\}$ be the free monoid generated by $\left\{X_{0}, \ldots, X_{p}\right\}$ and $\varphi: F \rightarrow S$ be the monoid epimorphism defined by

$$
\varphi\left(a_{0} X_{0}+\ldots+a_{p} X_{p}\right)=a_{0} n_{0}+\ldots+a_{p} n_{p} .
$$

It is known (see [7]) that if $\sigma$ is the kernel congruence of $\varphi$, which is defined by $x \sigma y$ if and only if $\varphi(x)=\varphi(y)$, then $S$ is isomorphic to the quotient monoid $F / \sigma$. Rédei proves in [6] that the congruence $\sigma$ is finitely generated and therefore there exists

$$
\rho=\left\{\left(x_{1}, y_{1}\right), \ldots,\left(x_{t}, y_{t}\right)\right\} \subseteq F \times F
$$

such that $\sigma$ is the smallest congruence on $F$ which contains $\rho$. The set $\rho$ is called a presentation for the numerical semigroup $S$. We say that a presentation is minimal if

Received by the editors July 29, 1999 and, in revised form, December 9, 1999.

2000 Mathematics Subject Classification. Primary 20M14, 20M05, 20M30, 13H10.

Key words and phrases. Symmetric numerical semigroup, multiplicity, embedding dimension.

This paper was supported by the project DGES PB96-1424.

The author would like to thank P. A. García-Sánchez, J. I. García-García and the referee for their comments and suggestions. 
there is not any proper subset of $\rho$ generating $\sigma$. In [8] it is shown that the concepts of minimal presentation and presentation with the lowest cardinality coincide for a numerical semigroup.

We say that a numerical semigroup $S$ is symmetric if every integer $z \notin S$ verifies that $\mathrm{C}(S)-z \in S$. It is known (see 9]) that if $\mathrm{m}(S) \geq 3$ and $S$ is symmetric, then $\mu(S) \leq \mathrm{m}(S)-1$. Our principal aim in this paper is to prove that if $m$ and $e$ are nonnegative integers such that $2 \leq e \leq m-1$, then there exists a symmetric semigroup $S$ with $\mathrm{m}(S)=m$ and $\mu(S)=e$. The proof that we give is constructive, thus we can obtain a family of symmetric semigroups with arbitrary multiplicity and embedding dimension. Furthermore, we show that if $\mu(S) \geq 3$, then the cardinality of any minimal presentation for all semigroups in this family is equal to

$$
\frac{\mu(S)(\mu(S)-1)}{2}-1 .
$$

From our point of view, this is an interesting result for two reasons. The first one is that determining from $m$ all the possible cardinals of minimal presentations for numerical semigroups with multiplicity $m$ is an open problem. From [10] it is deduced that the cardinality of a minimal presentation for a numerical semigroup with multiplicity equal to 5 can be $1,2,3,5,6$ or 10 . In 11 it is shown that if $e \in\{2, \ldots, m\}$, then there exists a numerical semigroup with multiplicity $m$ and with the cardinality of its minimal presentations equal to $e(e-1) / 2$. With the results of this work we can assert that if $e \in\{2, \ldots, m-1\}$ there exists a numerical semigroup, which is symmetric, has multiplicity $m$ and the cardinality of its minimal presentations is equal to $e(e-1) / 2-1$. The second one is that in [2, Bresinsky gives a family of numerical semigroups with embedding dimension equal to 4 and with the cardinality of its minimal presentations arbitrarily large. This proves that the cardinality of a minimal presentation for a numerical semigroup cannot be bounded as a function of its embedding dimension. Bresinsky also proves in [3] that the cardinality of a minimal presentation for a symmetric numerical semigroup with embedding dimension 4 can only be 3 or 5 . This makes us speculate about the possibility that the cardinality of a minimal presentation for a symmetric numerical semigroup can be bounded in a function of its embedding dimension and a candidate for it is

$$
\frac{\mu(S)(\mu(S)-1)}{2}-1
$$

if $\mu(S) \geq 3$. Hence, if this conjecture is true, the family of symmetric numerical semigroups presented in this work is very interesting because the bound is reached by this family.

In concluding this introduction, note that although this work is performed from a semigroupist point of view, it can be used in Ring Theory. If $\mathbb{K}$ is a field, $\mathbb{K}[S]$ is the finite type $\mathbb{K}$-algebra associated to $S$ and $\mathbb{K}[X]=\mathbb{K}\left[X_{0}, \ldots, X_{p}\right]$ is the polinomial ring in $p+1$ indeterminates, the $\mathbb{K}$-algebra epimorphism $\lambda: \mathbb{K}[X] \rightarrow \mathbb{K}[S]$ which takes $X_{i}$ to $t^{n_{i}}$ is an $S$-graduated ring homomorphism with degree zero. Therefore, the prime ideal $P=\operatorname{Kernel}(\lambda)$ (called the ideal associated to the semigroup) is homogeneous and defines a monomial curve in the $(p+1)$-dimensional affine space on $\mathbb{K}$. Herzog in 4 proved that finding a system of generators for $P$ is equivalent to finding a presentation for $S$. Finally, Kunz in [5] proved that $\mathbb{K}[S]$ is Gorenstein if and only if $S$ is symmetric. 


\section{TWO FAMILIES OF SYMMETRIC NUMERICAL SEMIGROUPS}

Let $S$ be a numerical semigroup and $n \in S \backslash\{0\}$. Denote by

$$
0=w(1)<w(2)<\ldots<w(n)
$$

the smallest elements of $S$ in respective congruences class $\bmod n$. We denote by $\operatorname{Ap}(S, n)$ (the Apéry set of $n$ in $S$ ) the set $\{0=w(1)<w(2)<\ldots<w(n)\}$. It is known (see 9]) that $w(n)=\mathrm{C}(S)+n$ and that $S$ is symmetric if and only if $w(i)+w(n-i+1)=w(n)$ for all $i \in\{1, \ldots, n\}$.

Lemma 1. Let $m$ and $q$ be positive integers such that $m \geq 2 q+3$ and $S$ be the submonoid of $(\mathbb{N},+)$ generated by

$$
\{m, m+1, q m+2 q+2, \ldots, q m+(m-1)\} .
$$

Then $S$ is a symmetric numerical semigroup with multiplicity $m$, embedding dimension $m-2 q$ and Frobenius number $2 q m+2 q+1$.

Proof. Since g.c.d. $\{m, m+1\}=1$, we have that $S$ generates $\mathbb{Z}$ as a group and therefore $S$ is a numerical semigroup. Clearly, $\{m, m+1, q m+2 q+2, \ldots, q m+$ $(m-1)\}$ is a minimal system of generators for $S$ and thus $\mathrm{m}(S)=m$ and $\mu(S)=$ $m-2 q$. It is easy to deduce that

$$
\begin{gathered}
\operatorname{Ap}(S, m)=\{0<m+1<2 m+2<\ldots<q m+q \\
<q m+2 q+2<\ldots<q m+(m-1) \\
<(q+1) m+q+1<\ldots<(2 q+1) m+2 q+1\} .
\end{gathered}
$$

For showing that $S$ is symmetric we use the characterization given before this Lemma. We need to prove that for all $w \in \operatorname{Ap}(S, m)$ there exists $w^{\prime} \in \operatorname{Ap}(S, m)$ such that $w+w^{\prime}=(2 q+1) m+2 q+1$.

- For $i \in\{0,1, \ldots, m-1-2 q-2\}$ we have that

$$
(q m+2 q+2+i)+(q m+m-1-i)=(2 q+1) m+2 q+1 .
$$

- For $k \in\{1,2, \ldots, q\}$ we obtain

$$
(k m+k)+((2 q+1-k) m+2 q+1-k)=(2 q+1) m+2 q+1 .
$$

Finally since $\mathrm{C}(S)+m=\operatorname{maximum}(\operatorname{Ap}(S, m))$, we deduce that $\mathrm{C}(S)=2 q m+2 q+$ 1.

Example 2. We take $m=12$ and $q=3$. Then we have that the submonoid of $(\mathbb{N},+)$ generated by $\{12,13,44,45,46,47\}$ is a symmetric numerical semigroup with multiplicity 12, embedding dimension 6 and Frobenius number 79.

Lemma 3. Let $m$ and $q$ be nonnegative integers such that $m \geq 2 q+4$ and $S$ be the submonoid of $(\mathbb{N},+)$ generated by

$$
\{m, m+1,(q+1) m+q+2, \ldots,(q+1) m+m-q-2\} .
$$

Then $S$ is a symmetric numerical semigroup with multiplicity $m$, embedding dimension $m-2 q-1$ and Frobenius number $2(q+1) m-1$. 
Proof. As in Lemma 1, we deduce that $S$ is a numerical semigroup with $\mathrm{m}(S)=m$ and $\mu(S)=m-2 q-1$.

It is easy to prove that

$$
\begin{gathered}
\operatorname{Ap}(S, m)=\{0<m+1<2 m+2<\ldots<(q+1) m+q+1 \\
<(q+1) m+q+2<\ldots<(q+1) m+m-q-2<(q+2) m+m-q-1 \\
<(q+3) m+(m-q)<\ldots<2(q+1) m+m-1\} .
\end{gathered}
$$

Furthermore, as a consequence of the following comments, $S$ is symmetric:

- For $i \in\{0,1, \ldots, m-2 q-3\}$,

$$
((q+1) m+q+1+i)+((q+1) m+m-q-2-i)=2(q+1) m+m-1 .
$$

- For $k \in\{0,1, \ldots, q\}$,

$$
((q+2+k) m+m-q-1+k)+((q-k) m+q-k)=2(q+1) m+m-1 .
$$

Finally, since $\mathrm{C}(S)+m=\operatorname{maximum}(\operatorname{Ap}(S, m))$, we obtain that $\mathrm{C}(S)=2(q+1) m-$ 1.

Example 4. Let $m=15$ and $q=4$. Thus we obtain the submonoid of $(\mathbb{N},+)$ generated by $\{15,16,81,82,83,84\}$. Using Lemma 3 , we know that it is a symmetric numerical semigroup with multiplicity 15, embedding dimension 6 and Frobenius number 149 .

Theorem 5. Let $m$ and $e$ be nonnegative integers such that $2 \leq e \leq m-1$. There exists a symmetric numerical semigroup with multiplicity $m$ and embedding dimension e.

Proof. If $e=2$, then $S=\langle m, m+1\rangle$ is a symmetric numerical semigroup with multiplicity $m$ and embedding dimension 2 (it is a known fact that all the numerical semigroups with embedding dimension equal to 2 are symmetric). Thus, in the sequel, we shall suppose $e \geq 3$. We distinguish two cases:

- If $m-e$ is even, then there exists $q \in \mathbb{N} \backslash\{0\}$ such that $m-e=2 q$. Furthermore, $e \geq 3$ implies that $m \geq m-e+3$ and therefore $m \geq 2 q+3$. Lemma 1 asserts the existence of a symmetric numerical semigroup with multiplicity $m$ and embedding dimension $e=m-2 q$.

- If $m-e$ is odd, then there exists $q \in \mathbb{N}$ such that $m-e=2 q+1$. Furthermore, $e \geq 3$ implies that $m \geq m-e+3$ and therefore $m \geq 2 q+4$. Lemma 3 implies the existence of a symmetric numerical semigroup with multiplicity $m$ and embedding dimension $e=m-2 q-1$.

We finish this paper by proving that if $S$ is a numerical semigroup obtained from Lemma 1 or Lemma 3, then the cardinality of a minimal presentation for $S$ is equal to

$$
\frac{\mu(S)(\mu(S)-1)}{2}-1 .
$$

Before proving that, we need to introduce some concepts and results.

If $S$ is a numerical semigroup with a minimal system of generators $\left\{n_{0}<\ldots<\right.$ $\left.n_{p}\right\}$ and $s \in S$, then there exists $\left(a_{0}, \ldots, a_{p}\right) \in \mathbb{N}^{p+1}$ such that $s=a_{0} n_{0}+\ldots+a_{p} n_{p}$. 
We say that an element $s$ has a unique expression if $\left(a_{0}, \ldots, a_{p}\right)$ is unique. We denote it by

$$
\operatorname{Ap}\left(S, n_{0}\right)=\left\{s \in S \mid s-n_{0} \notin S\right\} .
$$

In [11] it is given a method for obtaining a minimal presentation for a numerical semigroup fulfilling the condition that all the elements of $\operatorname{Ap}\left(S, n_{0}\right)$ have a unique expression. The process is as follows: Denote by

$$
T=\left\{\left(a_{1}, \ldots, a_{p}\right) \in \mathbb{N}^{p} \mid a_{1} n_{1}+\ldots+a_{p} n_{p} \notin \operatorname{Ap}\left(S, n_{0}\right)\right\}
$$

and

$$
\left\{\alpha_{1}=\left(\alpha_{11}, \ldots, \alpha_{1 p}\right), \ldots, \alpha_{t}=\left(\alpha_{t 1}, \ldots, \alpha_{t p}\right)\right\}=\operatorname{minimals}(T)
$$

with respect to the usual order of $\mathbb{N}^{p}$ (note that by Dickson's Lemma this set is finite). For every $i \in\{1, \ldots, t\}$ we define $x_{i}=0 X_{0}+\alpha_{i 1} X_{1}+\ldots+\alpha_{i p} X_{p} \in F$. Since $\varphi\left(x_{i}\right) \notin \operatorname{Ap}\left(S, n_{0}\right)$, we deduce that there exists $\left(\beta_{i 0}, \beta_{i 1}, \ldots, \beta_{i p}\right) \in \mathbb{N}^{p+1}$ with $\beta_{i 0} \neq 0$ such that

$$
\varphi\left(x_{i}\right)=\beta_{i 0} n_{0}+\beta_{i 1} n_{1}+\ldots+\beta_{i p} n_{p} .
$$

For every $i \in\{1, \ldots, t\}$, we define $y_{i}=\beta_{i 0} X_{0}+\ldots+\beta_{i p} X_{p}$. Note that $\varphi\left(x_{i}\right)=\varphi\left(y_{i}\right)$ for all $i \in\{1, \ldots, t\}$ and thus

$$
\rho=\left\{\left(x_{1}, y_{1}\right), \ldots,\left(x_{t}, y_{t}\right)\right\} \subseteq \sigma .
$$

In [11] the following result is proved.

Theorem 6. Let the hypothesis be as above. Then

$$
\rho=\left\{\left(x_{1}, y_{1}\right), \ldots,\left(x_{t}, y_{t}\right)\right\}
$$

is a minimal presentation for $S$.

Now, we can to compute the cardinality of the minimal presentations for the semigroups obtained from Lemma 1 and Lemma 3.

Proposition 7. Under the hypothesis of Lemma 1. The cardinality of a minimal presentation for the numerical semigroup $S$ generated by

$$
\{m, m+1, q m+2 q+2, \ldots, q m+(m-1)\}
$$

is equal to

$$
\frac{\mu(S)(\mu(S)-1)}{2}-1 .
$$

Proof. Setting $n_{0}=m, n_{1}=m+1, n_{2}=q m+2 q+2, \ldots, n_{p}=q m+(m-1)$, we obtain that

$$
\begin{gathered}
\operatorname{Ap}\left(S, n_{0}\right)=\left\{0<n_{1}<2 n_{1}<\ldots<q n_{1}<n_{2}<\ldots<n_{p}<(q+1) n_{1}\right. \\
<(q+2) n_{1}<\ldots<2 q n_{1}<(2 q+1) n_{1}=n_{2}+n_{p} \\
\left.=n_{3}+n_{p-1}=\ldots=n_{[(p+2) / 2]}+n_{[(p+3) / 3]}\right\}
\end{gathered}
$$

where $[x]$ denotes the integer part of $x$. Note that all the elements, except the maximum of $\operatorname{Ap}\left(S, n_{0}\right)$, have a unique expression. In [9], it is proved that in this setting $S^{\prime}=S \cup\{\mathrm{C}(S)\}$ is a numerical semigroup with a minimal system of generators

$$
\left\{n_{0}<n_{1}<\ldots<n_{p}<n_{p+1}=\mathrm{C}(S)\right\} .
$$


Moreover, if

$$
\operatorname{Ap}\left(S, n_{0}\right)=\{0=w(1)<w(2)<\ldots<w(n-1)<w(n)\},
$$

then

$$
\operatorname{Ap}\left(S^{\prime}, n_{0}\right)=\{0=w(1)<w(2)<\ldots<w(n-1)<\mathrm{C}(S)\}
$$

furthermore, if $\rho$ and $\rho^{\prime}$ are minimal presentations for $S$ and $S^{\prime}$ respectively, then $\# \rho^{\prime}=\# \rho+p+2($ where $\# A$ denotes the cardinality of the set $A)$.

Using the above remark we can assert that

$$
\operatorname{Ap}\left(S^{\prime}, n_{0}\right)=\left\{0, n_{1}, 2 n_{1}, \ldots, q n_{1}, n_{2}, \ldots, n_{p},(q+1) n_{1}, \ldots, 2 q n_{1}, n_{p+1}\right\} .
$$

Clearly, all the elements of $\operatorname{Ap}\left(S^{\prime}, n_{0}\right)$ have a unique expression. Applying Theorem 6] we deduce that a minimal presentation for $S^{\prime}$ is

$$
\begin{gathered}
\rho^{\prime}=\left\{\left((2 q+1) X_{1}, y_{1}\right),\left(X_{1}+X_{2}, y_{2}\right), \ldots,\left(X_{1}+X_{p+1}, y_{p+1}\right),\right. \\
\left(2 X_{2}, y_{p+2}\right),\left(X_{2}+X_{3}, y_{p+3}\right), \ldots,\left(X_{2}+X_{p+1}, y_{p+1+p}\right),\left(2 X_{3}, y_{p+1+p+1}\right), \\
\left(X_{3}+X_{4}, y_{p+1+p+2}\right) \ldots,\left(X_{3}+X_{p+1}, y_{p+1+p+p-1}\right), \ldots,\left(2 X_{p}, y_{(p+1)+p+\ldots+3+1}\right), \\
\left.\left(X_{p}+X_{p+1}, y_{(p+1)+p+\ldots+3+2}\right),\left(2 X_{p+1}, y_{p+1+\ldots+p+3+2+1}\right)\right\},
\end{gathered}
$$

where if $(x, y) \in \rho^{\prime}, \varphi(x)=\varphi(y)$ and $y$ is of the form

$$
a_{0} X_{0}+\ldots+a_{p} X_{p}+a_{p+1} X_{p+1}
$$

with $a_{0} \neq 0$. In particular, we have that

$$
\# \rho^{\prime}=\frac{(p+1)(p+2)}{2}
$$

Since $\# \rho^{\prime}=\# \rho+p+2$, we have that

$$
\# \rho=\frac{p(p+1)}{2}-1=\frac{\mu(S)(\mu(S)-1)}{2}-1
$$

Proposition 8. Under the hypothesis of Lemma 3. Then the cardinality of a minimal presentation for the semigroup $S$ generated by

$$
\{m, m+1,(q+1) m+q+2, \ldots,(q+1) m+m-q-2\}
$$

is equal to

$$
\frac{\mu(S)(\mu(S)-1)}{2}-1
$$

Proof. Setting $n_{0}=m, n_{1}=m+1, n_{2}=(q+1) m+q+2, \ldots, n_{p}=(q+1) m+$ $m-q-2$, we have that

$$
\begin{gathered}
\operatorname{Ap}\left(S, n_{0}\right)=\left\{0<n_{1}<2 n_{1}<\ldots<(q+1) n_{1}<n_{2}<\ldots<n_{p}<n_{p}+n_{1}\right. \\
<n_{p}+2 n_{1}<\ldots<n_{p}+q n_{1}<n_{p}+(q+1) n_{1}=n_{2}+n_{p-1} \\
\left.=n_{3}+n_{p-2}=\ldots=n_{[(p+1) / 2]}+n_{[(p+2) / 2]}\right\} .
\end{gathered}
$$

All the elements of $\operatorname{Ap}\left(S, n_{0}\right)$, except the maximum, have a unique expression. Thus the numerical semigroup $S^{\prime}=S \cup\{\mathrm{C}(S)\}$ has a minimalsystem of generators 


$$
\begin{aligned}
& \left\{n_{0}<n_{1}<\ldots<n_{p}<n_{p+1}=\mathrm{C}(S)\right\} ; \text { furthermore } \\
& \qquad \begin{array}{c}
\operatorname{Ap}\left(S^{\prime}, n_{0}\right)=\left\{0, n_{1}, 2 n_{1}, \ldots,(q+1) n_{1}, n_{2}, \ldots, n_{p},\right. \\
\left.n_{p}+n_{1}, n_{p}+2 n_{1}, \ldots, n_{p}+q n_{1}, n_{p+1}\right\}
\end{array}
\end{aligned}
$$

and all its elements have a unique expression. Applying Theorem [6, we deduce that a minimal presentation for $S^{\prime}$ is

$$
\begin{gathered}
\rho^{\prime}=\left\{\left((q+2) X_{1}, y_{1}\right),\left(X_{1}+X_{2}, y_{2}\right), \ldots,\left(X_{1}+X_{p-1}, y_{p-1}\right),\right. \\
\left((q+1) X_{1}+X_{p}, y_{p}\right),\left(X_{1}+X_{p+1}, y_{p+1}\right),\left(2 X_{2}, y_{p+2}\right), \\
\left(X_{2}+X_{3}, y_{p+3}\right), \ldots,\left(X_{2}+X_{p+1}, y_{p+1+p}\right),\left(2 X_{3}, y_{p+1+p+1}\right), \\
\left(X_{3}+X_{4}, y_{p+1+p+2}\right), \ldots,\left(X_{3}+X_{p+1}, y_{p+1+p+p-1}\right), \ldots,\left(2 X_{p}, y_{p+1+p+\ldots+3+1}\right), \\
\left.\left(X_{p}+X_{p+1}, y_{p+1+p+\ldots+3+2}\right),\left(2 X_{p+1}, y_{p+1+p+\ldots+3+2+1}\right)\right\} .
\end{gathered}
$$

Hence, we obtain that $\# \rho^{\prime}=\frac{(p+1)(p+2)}{2}$. Applying that if $\rho$ is a minimal presentation for $S$, then

$$
\# \rho^{\prime}=\# \rho+p+2
$$

we deduce that

$$
\# \rho=\frac{\mu(S)(\mu(S)-1)}{2}-1
$$

\section{REFERENCES}

[1] V. Barucci, D. E. Dobbs and M. Fontana, "Maximality Properties in Numerical Semigroups and Applications to One-Dimensional Analyticalle Irreducible Local Domains", Memoirs of the Amer. Math. Soc. 125(1997). MR 97g:13039

[2] H. Bresinsky, On prime ideal with generic zero $x_{i}=t^{n_{i}}$, Proc. Amer. Math. Soc. 47(1975) 329-332. MR 52:10741

[3] H. Bresinsky, Symmetric semigroups of integers generated by 4 elements, Manuscripta Math. 17(1975) 205-219. MR 54:2660

[4] J. Herzog, Generators and relations of abelian semigroups and semigroup rings, Manuscripta Math. 3(1970), 175-193. MR 42:4657

[5] E. Kunz, The value semigroup of an one dimensional Gorenstein ring, Proc. Amer. Math. Soc. 25(1970), 748-751. MR 42:263

[6] L. Rédei, "The theory of finitely generated commutative semigroups." Pergamon, OxfordEdinburgh-New York, 1965. MR 32:5761

[7] J. C. Rosales and P. A. García-Sánchez, "Finitely generated commutative monoids", Nova Science Publishers, NewYork, 1999. MR 2000d:20074

[8] J. C. Rosales, An algorithm for determining a minimal relation associated to a numerical semigroup, Int. J. Algebra and Comput. 6(1996), 441-455. MR 97f:20080

[9] J. C. Rosales, On symmetric numerical semigroups, J. Algebra 182(1996), 422-434. MR 98h:20099

[10] J. C. Rosales and P. A. García-Sánchez, On numerical semigroups with high embedding dimension, J. Algebra 203(1998), 567-578. MR 99g:20108

[11] J. C. Rosales, Numerical Semigroups with Apéry sets of unique expression, J. Algebra 226 (2000), 479-487.

Departamento de Álgebra, Universidad de Granada, E-18071 Granada, Spain

E-mail address: jrosales@ugr.es 